\title{
MAPEAMENTO DA COBERTURA VEGETAL A PARTIR DE IMAGENS DE ALTA RESOLUÇÃO OBTIDAS POR VANT
}

\author{
Filipe Castro Felix ${ }^{(a)}$, Fabio Arnaldo Pomar Avalos ${ }^{(b)}$, Marx Leandro Naves Silva ${ }^{(c)}$, Ronaldo \\ Luiz Mincato $^{(\mathrm{d})}$
}

(a) PPGCA/Universidade Federal de Alfenas - UNIFAL-MG, filipecfelix@ gmail.com

(b) DCS/ Universidade Federal de Lavras - UFLA, fa.ingeo@ @mail.com

(c) DCS/ Universidade Federal de Lavras - UFLA, marx @ dcs.ufla.br

(d) PPGCA/Universidade Federal de Alfenas - UNIFAL-MG, ronaldo.mincato@ unifal-mg.edu.br

\section{Eixo: GEOTECNOLOGIAS E MODELAGEM ESPACIAL EM GEOGRAFIA FÍSICA}

\begin{abstract}
Resumo
Veículos Aéreos Não Tripulados ou Drones estão em uso crescente nos estudos ambientais, permitindo a coleta dados precisos para mapeamento e monitoramento da paisagem. Contudo, foram pouco explorados nos estudos de cobertura vegetal e monitoramento florestal. Portanto, esta pesquisa visou mapear a cobertura vegetal de uma área experimental com: Drone Phantom 3 Professional, 150 pontos de classificação da vegetação e 4 índices de cobertura vegetal, no espectro visível. Para tanto, foram realizados levantamentos de campo, para validação do aerolevantamento. Os resultados demonstraram a necessidade de melhorar os estudos dos índices de vegetação no visível, pois foi detectada dificuldades de distinção dos diferentes tipos de vegetação da área pelos índices selecionados. Assim, as alternativas para resolução do problema seriam: avaliação sazonal das respostas espectrais, uso de sensores infravermelhos e novos índices do espectro visível e do visível conjugado com o infravermelho, para validação do método de estudo.
\end{abstract}

Palavras-chave: VANT - solos - agricultura de precisão - remanescentes florestais - conservação

\section{Introdução}

O solo é um recurso natural não renovável na escala de tempo humana, cuja sustentabilidade é vital para manutenção da humanidade. De acordo com Stoffel e Huggel (2012), um dos maiores problemas ambientais da atualidade é a degradação dos solos, uma vez que seu processo de formação é lento, complexo e diretamente afetado pelas atividades antrópicas (WALKER, 1994).

No Brasil, as pesquisas voltadas às ciências do solo foram tardias, só ganhando impulso a partir da década de 1970, junto às políticas públicas de desenvolvimento, que estimularam o avanço das fronteiras agrícolas para regiões inexploradas. Desta forma, este período foi marcado por elevados índices de desmatamentos, causados, em primeiro lugar, pelo crescimento da malha rodoviária, como a Transamazônica (FEARNSIDE, 2005; MITTERMEIER et al., 2005; FEARNSIDE, 2007), e, em segundo, pelas atividades agropecuárias extensivas e intensivas (BEZERRA; CLEPES JÚNIOR, 2004; 
RODRIGUES; MIAZIARA, 2008), que associadas à falta de manejo dos solos e de práticas conservacionistas adequadas, como, por exemplo, o plantio direto, são as principais responsáveis pela degradação, aumentando as pressões sobre novas áreas (SOS MATA ATLÂNTICA; INPE, 2013).

Atualmente, os remanescentes florestais representam fragmentos de uma paisagem modificada pelo homem, que é o panorama ideal para gerar mudanças na estrutura e composição das comunidades biológicas ao longo dos anos (LAURANCE et al., 2011). A redução da quantidade, do tamanho e o isolamento destes fragmentos são os responsáveis pela perda da biodiversidade (CALEGARI et al., 2010), alteração do controle geobiohidrológico (CHECCIA; GUINDANI, 2003; KOBYIAMA, 2003; HINKEL, 2003), do sequestro de carbono, da manutenção da temperatura e do microclima (ARROJA et al., 2006) e, também, da perda de conexão entre os habitats por corredores ecológicos (METZGER, 2010), que viabilizam o fluxo gênico das populações (JACOB, 2003). Portanto, pesquisas que permitam indicar ações rápidas e precisas para melhorar a gestão e o planejamento da ocupação do território são necessárias, pois ajudam a propor medidas de mitigação de impactos e de conservação dos solos e da diversidade biológica.

Neste cenário, o sensoriamento remoto (SR) associado aos sistemas de informação geográfica (SIG) pode complementar as técnicas de estudo de campo a partir da captura de informações em diversas escalas temporais, espectrais e espaciais, tornando o monitoramento e investigação da paisagem mais eficazes (GÓMEZ, et al., 2012). Não obstante, contribui ainda com economia de recursos e de tempo (YUAN et al., 2005; ZHANG; WANG et al., 2011), que justificam o uso recorrente desta ferramenta nos estudos de avaliação da cobertura florestal (HANSEN et al., 2013; NIJLAND et al., 2015), de densidade de árvores (CROWTHER et al., 2015), de distribuição de espécies (CORD et al., 2013), do dossel (SIMARD et al., 2011; NIJLAND et al., 2015; ZHANG et al., 2016) e de reservas de carbono (SAATCHI et al., 2011; ZHANG et al., 2014). No entanto, as tecnologias convencionais, como os satélites, estão perdendo espaço devido a muitos fatores, como o acesso restrito, o alto custo de obtenção, operação e limitações de seus produtos. Dentre as limitações, cabe destacar a falta de resolução temporal para as pesquisas, já que o intervalo entre os registros é, em média, 16 dias, além da contaminação das imagens por nuvens, principalmente nas florestas tropicais e subtropicais, que inviabilizam muitas propostas de pesquisa (PANEQUE-GÁLVEZ et al., 2014; WHITEHEAD et al., 2014).

Diante disso, uma ferramenta promissora que está ganhando aplicações civis são os Veículos Aéreos Não Tripulados (VANT) ou Drones. Com surgimento junto ao Geographic Position System (GPS) para usos militares (NEWCOME, 2004), eles apresentam boa acurácia em estudos ambientais, sendo muito utilizados na agricultura de precisão e no monitoramento de habitats, pois permitem a coleta de dados em curto espaço de tempo. Outras vantagens apresentadas, são a sua capacidade de sobrevoar áreas em baixas 
altitudes $(30-300 \mathrm{~m})$ e, consequentemente, sem interferência das nuvens, que os capacita a capturar imagens em alta resolução e a coletar dados em locais de difícil acesso (SILVA et al., 2015). Estas características promissoras permitem um alavancamento dos estudos de mapeamento e de monitoramento ambiental, porém algumas desvantagens como o tempo de voo curto e a necessidade de condições climáticas favoráveis, implicam, ainda, na pequena adesão desta plataforma para os estudos ambientais (GETZIN, 2012).

Apesar destas novas tecnologias estarem em pleno desenvolvimento, têm sido pouco aplicadas em estudos florestais (TREUHAFT et al., 2004). Segundo Tomlins (1983), o grande avanço dos VANT proporcionou ao menos 46 aplicações em estudos ambientais. No entanto, esta tecnologia permanece em desuso para a maioria dessas finalidades (HARDIN; HARDIN, 2010), inclusive nas pesquisas de cobertura vegetal. Assim, metodologias como as propostas por Stocking (1988) e Wischmeier e Smith (1978) continuam como os modelos mais aplicados no Brasil (BARRETO et al., 2010).

Esta pesquisa visou mapear a cobertura vegetal de uma parcela experimental no Município de Lavras, no sul de Minas Gerais, a partir da aplicação de índices de cobertura vegetal e imagens com alta resolução espacial obtidas a partir de um quadricóptero.

\section{Materiais e Métodos}

A área experimental ocupa aproximadamente 10 hectares da Fazenda Muquém, do Centro de Desenvolvimento Científico e Tecnológico na Agropecuária da Universidade Federal de Lavras, no Município de Lavras, Minas Gerais, que por sua vez cobre 160 hectares entre as Coordenadas UTM/SIRGAS 200023 K 500.591 m E; 7.655 .088 m N e 502.485 m E; 7656456 m N (figura 1).

O clima, segundo Dantas (2007), é o CwA, na classificação climática de Köppen, que se caracteriza como temperado chuvoso (mesotérmico) e inverno seco, subtropical com temperatura média anual de $19,4^{\circ}$ C. A altitude média é $918 \mathrm{~m}$, com precipitação média anual de 1.529,7 mm. Em relação à vegetação, a mesma é classificada como subperenefólia, representada, atualmente, por pequenos fragmentos dispersos. A propriedade apresenta predominância das lavouras de feijão e pastagens naturais, além de uma área destinada à silvicultura.

Os solos da área são classificados em Neossolos, Gleissolos, Cambissolos, Argissolos, Nitossolos e Latossolos, com a predominância do Latossolo Vermelho-Amarelo e do Cambissolo Háplico argissólico (SILVA et al., 2014). 


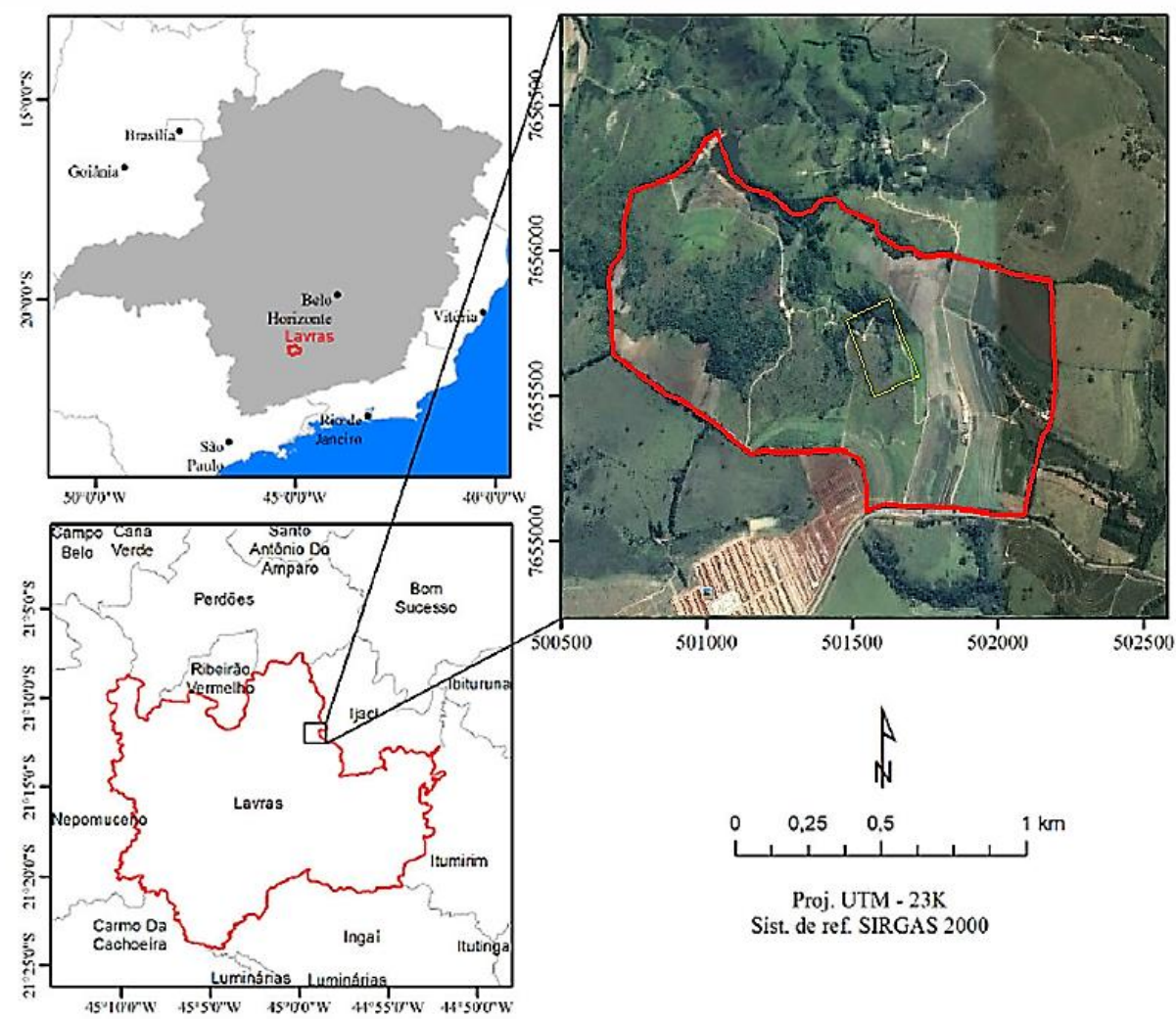

Figura 1 - Localização da Fazenda Muquém (Centro de Desenvolvimento Científico e Tecnológico na Agropecuária da Universidade Federal de Lavras), com destaque à area experimental (em amarelo), no Município de Lavras, Minas Gerais.

Em síntese, a proposta de estudo é separada em três etapas: (1) levantamentos com Drone, (2) levantamentos de campo e (3) aplicação dos índices de vegetação. Com base nas técnicas apontadas, o objetivo é definir a mais eficaz para o mapeamento da cobertura vegetal.

Na primeira etapa foi utilizado o VANT Phantom 3, versão Professional, que pesa $1.280 \mathrm{~g}$, e é embarcado com uma câmera de modelo Sony EXMOR 1/2.3", com total de pixels efetivos 12,76 M, que permite capturar imagens em cores reais de tamanho máximo de 4.000 por 3.000 pixels nas bandas R, G e B (Red, Green e Blue), com uma lente FOV $94^{\circ} 20 \mathrm{~mm}$. Este equipamento multirrotor pode voar por controle remoto ou de forma autônoma, cumprindo planos de navegação pré-definidos por GPS.

O voo foi planejado na altitude de 90 metros para o mês de fevereiro de 2017. Logo, o mapeamento da área consistiu em 1 voo durante o verão para extrair os dados de reflectância das diferentes coberturas vegetais.

O planejamento do voo foi a partir do software Pix4D, responsável por determinar os pontos de passagem, assim como os níveis de sobreposição das imagens, tanto de overlap, como de sidelap, que respeitaram o 


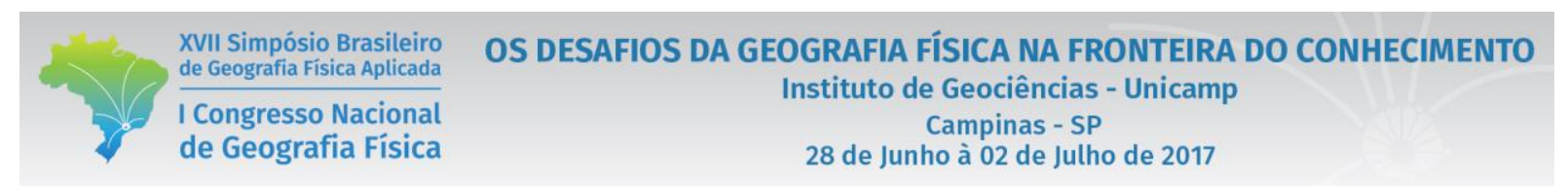

mínimo de $60 \%$ para garantir a qualidade dos dados do levantamento aerofotogramétrico. Para processar os produtos do Drone, foi aplicado o software Agisoft Photoscan, que embora não seja um software livre é o mais utilizado para o processamento e a obtenção dos ortomosaicos georreferenciados (TORRESSÁNCHEZ et al., 2014). Na etapa seguinte, na área experimental foram definidos 150 pontos aleatórios, que posteriormente foram classificados em: (i) vegetação nativa, (ii) vegetação em regeneração, (iii) pasto, (iv) várzea e (v) solos expostos, de acordo com os dados mapeados previamente em campo e análise das imagens via Drone.

Na segunda etapa, foi feita a extração e tratamento dos dados de cobertura vegetal, pelo software ArcGIS 10.1, em que foram avaliados 4 índices de vegetação: GMR (WANG et al., 2013), Excess Green (ExG) (WOEBBECKE et al., 1995), Kindex (KI) (KAWASHIMA, NAKATANI, 1998) e NDI (MAO et al., 2003). Tais índices foram utilizados para mapear a cobertura vegetal por unidade de área e discriminar os usos da terra previamente levantados em campo.

A última etapa, consistiu na validação dos índices de vegetação aplicados, quantificando os intervalos de frequência da reflectância específicos para cada um dos 5 tipos de cobertura vegetal para determinar qual deles foi o mais efetivo no mapeamento da área.

\section{Resultados e Discussão}

Foram processadas 82 fotos obtidas no voo, com sobreposição de $70 \%$ e foi gerado um ortomosaico com resolução espacial de $3,53 \mathrm{~cm} /$ pixel, que permitiram confirmar a boa qualidade do aerolevantamento a partir da individualização de alguns alvos e até possíveis identificações botânicas, como foi o caso das palmeiras presentes na área. Sendo assim, foram distribuídos 150 pontos aleatórios de mapeamento e classificação da cobertura vegetal, sendo 56 deles de pastagem, 41 de vegetação nativa, 31de vegetação em regeneração, 20 de solo exposto e 2 de várzea (figura 2). 


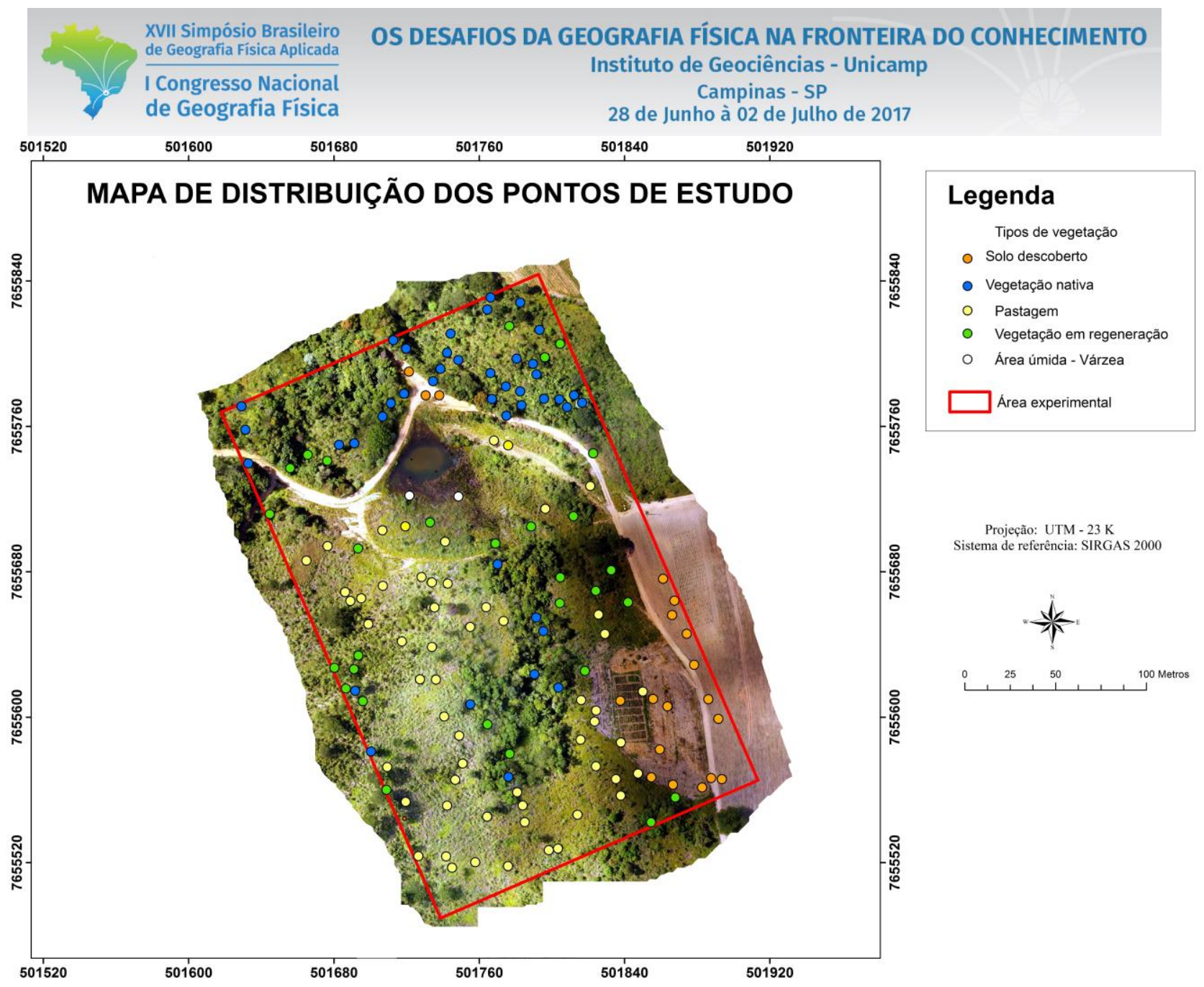

Figura 2 - Distribuição dos pontos aleatórios de classificação da cobertura vegetal.

Em seguida, os 4 índices de vegetação estudados foram calculados individualmente para cada ponto, ou seja, foi obtida a distribuição de frequência da reflectância de cada ponto e para cada classe de cobertura vegetal mapeada. Portanto, nesta etapa foi inicialmente determinado o valor de distinção entre as áreas com vegetação e sem vegetação e, posteriormente, foram definidos os intervalos de classificação de pastagem, vegetação nativa, vegetação em regeneração, solo exposto e várzea. Assim, o índice GMR apresentou o valor mais elevado de distinção que foi 25, seguido pelo Kindex com 0,57; o NDI com 0,31 e o Excess Green com 0,08. Contudo, todos os quatro índices apresentaram inconsistências na classificação das coberturas estudadas, visto que ocorreram sobreposições principalmente nos intervalos das classes de pastagem e vegetação em regeneração (Tabela I). 


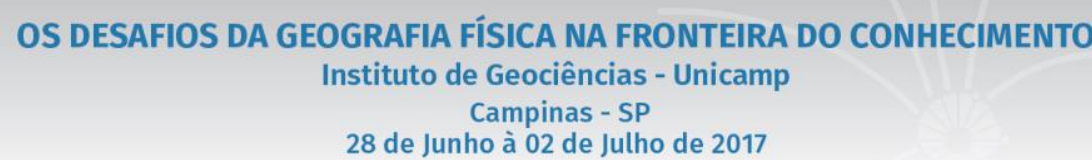

Tabela I - Distribuição das frequências de reflectância dos 4 índices de cobertura vegetal em relação aos 5 tipos de vegetação.

\begin{tabular}{lcccc}
\hline Tipos de vegetação & \multicolumn{4}{c}{ Índices de cobertura vegetal } \\
\cline { 2 - 4 } & \multicolumn{1}{c}{ Kindex } & NDI & GMR & Exg \\
\cline { 2 - 5 } Pastagem & $-0,137+-0,908$ & $0,072+0,891$ & $26+112$ & $0,001+0,409$ \\
Vegetação nativa & $-0,311 \vdash-0,825$ & $0,315+0,825$ & $61+74$ & $0,201+0,506$ \\
Vegetação em & $-0,210 \vdash-0,737$ & $-0,203+-0,751$ & $31+52$ & $0,123+0,487$ \\
regeneração & & & & \\
Várzea & $-0,346 \vdash-0,456$ & $-0,279+-0,282$ & $35+54$ & $-0,016+0,113$ \\
Solo exposto & $-0,125 \vdash-0,476$ & $-0,105+-0,314$ & $45+52$ & $0,055+0,126$ \\
\hline
\end{tabular}

A partir da correlação entre os dados obtidos na área estudada foi verificado a necessidade de buscar alternativas para aplicação de índices de cobertura vegetal no estudo e monitoramento de áreas no espectro visível. Pois, representa uma zona de frequência de reflectância restrita e seus índices são baseados em variações do comprimento de onda próximas do verde. Tais fatores na área em estudo, que está localizada na zona de transição entre os biomas Cerrado e Mata Atlântica, e que é caracterizada pela vegetação rasteira e arbustiva entre os remanescentes florestais (EMBRAPA, 2014), explicam as sobreposições entre os intervalos de comprimento de onda de pastagem com vegetação em regeneração e várzea.

Outro fator a ser considerado é que a análise temporal da área poderia reduzir estes erros, visto que levantamentos em estações com menor intensidade de radiação como inverno, primavera e outono, influenciariam na melhor discriminação das coberturas por esses índices, corrigindo as diferenças já observadas durante o verão. Por outro lado, esta menor incidência de radiação solar poderá dificultar ainda mais a distinção dos diferentes tipos de cobertura vegetal a partir dos índices de vegetação considerados.

Desta forma, se destaca a importância dos sensores infravermelhos acoplados em Drones e outras plataformas de coleta de dados com alta resolução, pois permitem não só a melhor distinção e classificação da vegetação como também favorecem o monitoramento destas áreas a partir do acompanhamento fenológico das espécies. Contudo, como estes sensores de plataformas remotas ainda representam uma alternativa pouco acessível, os levantamentos de campo associados aos sensores tradicionais no espectro visível ainda são uma alternativa de baixo custo operacional e com resultados satisfatórios. Assim, o uso combinado das duas ferramentas pode auxiliar na melhor gestão do território, favorecendo a conservação e estudo destas regiões que são hotspots de biodiversidade e estão ameaçadas pela expansão antrópica.

Diante do exposto, existem as seguintes alternativas para atingir o objetivo de mapear as diferentes coberturas vegetais: (i) avaliar a resposta dos índices aqui considerados com as variações sazonais, apesar 
da diminuição da incidência de radiação solar nas estações outono, inverno e primavera; (ii) buscar e desenvolver novos índices de vegetação no espectro visível que possam superar os índices testados e (iii) acoplar sensores infravermelhos nos VANT, que adicionará um maior espectro de comprimentos de onda que favorecem a discriminação vegetal, inclusive no sensoriamento por satélites remotos e que permitirá até mesmo buscar novos índices, tanto no infravermelho como em correlações do infravermelho com o visível. A princípio, as duas últimas alternativas podem contribuir para diminuição das necessidades de trabalhos de campo, tornando os resultados mais precisos com a combinação da alta resolução espacial com o maior intervalo de comprimento de onda.

\section{Considerações finais}

O desenvolvimento da pesquisa possibilitará determinar o potencial dos VANT no estudo e monitoramento da cobertura vegetal em relação aos métodos tradicionais com sensores remotos orbitais. Esta aplicação é ainda pouco explorada no Brasil, mas já apresentou resultados iniciais promissores nesta pesquisa iniciada recentemente e já garantiu resultados satisfatórios em pesquisas europeias.

\section{Agradecimentos}

Agradecemos à CAPES (Comissão de Aperfeiçoamento de Pessoal do Nível Superior) pelas bolsas de Mestrado pelo Programa de Pós-Graduação em Ciências Ambientais (PPGCA) e Doutorado pelo Departamento de Ciências do Solo (DCS).

\section{Bibliografia}

ARROJA, L.; DIAS, A. C.; CAPELA, I. The role of Eucalyptus globulus forest and products in carbon sequestration. Climatic Change, v. 74, n. 1, p. 123-140, 2006.

BARRETO, V.C.M. et al. Índice de cobertura vegetal e sua modelagem para cultivares de soja no sul de Minas Gerais. Ciência e Agrotecnologia, p. 1168-1175, 2010.

BEZERRA, L. M. C.; CLEPS JUNIOR, J. O desenvolvimento agrícola da região centro-oeste e as transformações no espaço agrário do Estado de Goiás. Caminhos de Geografia, Uberlândia, v. 2, n. 2, p. 29-49, 2004.

CALEGARI, L. et al. Análise da dinâmica de fragmentos florestais no município de Carandaí, MG, para fins de restauração florestal. Revista Árvore, Viçosa-MG, v.34, n.5, p.871- 880, 2010.

CHECCIHA, T; GUINDANI, A.A. Sustentabilidade ambiental na zona ripária: uma abordagem emergética. I Seminário de Hidrologia Florestal: Zonas Ripárias - Alfredo Wagner/SC - 22/09/2003. p. 147 
CORD, A.F. et al.. Modelling species distributions with remote sensing data: bridging disciplinary perspectives. J. Biogeogr. 40, 2226-2227, 2013.

CROWTHER, T.W. et.al., 2015. Mapping tree density at a global scale. Nature 525, 201-205.

EMBRAPA. Solos para todos, perguntas e respostas - Embrapa, novembro, 2014.

FEARNSIDE, P.M., 2005. Deforestation in Brazilian Amazonia: history, rates, and consequences. Conservation Biology 19,680-688.

FEARNSIDE, P.M., 2007. Brazil' s Cuiaba-Santarem (BR-163) Highway: the environmental cost of paving a soy bean Corridor through the amazon. Environmental Management 39, 601-614.

FUNDAÇÃO SOS MATA ATLANTICA \& INSTITUTO NACIONAL DE PESQUISAS ESPACIAIS - INPE. Atlas dos Municípios da Mata Atlântica - período 2011-2012, Anexo 1. São Paulo, INPE, 2013. 100p.

GETZIN, S.; WIEGAND, K.; SCHÖNING, I. Assessing biodiversity in forests using very high-resolution images and unmanned aerial vehicles. Methods in Ecology and Evolution, v. 3, n. 2, p. 397-404, 2012

GÓMEZ, C.; et al. Modeling forest structural parameters in the mediterranean pines of central Spain using QuickBird-2 imagery and classification and regression tree analysis (CART). Remote Sens. 2012, 4, 135-159.

HANSEN,M.C., et al. 2013. High-resolution global maps of 21st-century forest cover change. Science 342, 850853.

HARDIN, P.J.; HARDIN, T.J. Small-Scale Remotely Piloted Vehicles in Environmental Research. Geogr. Compass 2010, 4, 1297-1311.

HINKEL, R. Vegetação ripária: funções e ecologia. I Seminário de Hidrologia Florestal: Zonas Ripárias - Alfredo Wagner/SC -2003. p. 40-46.

JACOB, A. D. Zonas ripárias: relações com a fauna silvestre. I Seminário de Hidrologia Florestal: Zonas Ripárias Alfredo Wagner/SC -2003. p. 112 e 119

KAWASHIMA, S; NAKATANI, M. An algorithm for estimating chlorophyll content in leaves using a video camera. Annals of Botany, v. 81, n. 1, p. 49-54, jan. 1998.

KOBYIAMA, M. Conceitos de zona ripária e seus aspectos geobiohidrológicos. I Seminário de Hidrologia Florestal: Zonas Ripárias - Alfredo Wagner/SC - 2003. p. 1-13 
LAURANCE, W. F. et al. The fate of Amazonian forest fragments: a 32-year investigation. Biological Conservation, v. 144, n. 1, p. 56-67, 2011.

MAO, W.; WANG, Y.; WANG, Y. Real-time detection of between-row weeds using machine vision. 2003 , St. Joseph, MI: American Society of Agricultural and Biological Engineers, 2003.

METZGER, J.P. O código florestal tem base científica? J.P. Metzger, Conservação e Natureza - Universidade de São Paulo - 2010. 17p.

MITTERMEIER, R.A. et al. A brief history of biodiversity conservation in Brazil. Conservation Biology 19, 601607,2005

NEWCOME, L.R. Unmanned Aviation: A Brief History of Unmanned Aerial Vehicles; American Institute of Aeronautics and Astronautics, Inc.: Reston, VA, USA, 2004.

NIJLAND, W. et. al.Comparing patterns in forest stand structure following variable harvests using airborne laser scanning data. For. Ecol. Manag. 354, 272-280, 2005.

RODRIGUES, D. M. T.; MIZIARA, F. Expansão da fronteira agrícola: a intensificação da pecuária bovina no estado de Goiás. Pesquisa Agropecuária Tropical, Goiânia, v. 38, n. 1, p. 14-20, 2008

SAATCHI, S. et al.Seeing the forest beyond the trees. Glob. Ecol. Biogeogr. 24, 606-610, 2005.

SILVA, B. M.; SANTOS, W. J. R.; MARQUES, J. J. G. S. M . Levantamento detalhado dos solos da Fazenda Muquém/UFLA, Lavras-MG. Lavras: UFLA, 2014 (Boletim Técnico).

SILVA, J. M. C., C. UHL \& G. MURRAY; 1996. Plant succession, landscape management, and The ecology of frugivorous birds in abandoned Amazonian pastures. Conservation Biology 10: 491-503.

SIMARD, M., et al. Mapping forest canopy height globally With spaceborne lidar. J. Geophys. Res. $116,2011$.

STOCKING, M.A. Assessing vegetative cover and management effect. In: LAL, R. (Ed.). Soil erosion research methods. Iowa: Soil and Water Conservation Society, p.163-167, 1988.

STOFFEL, M. et al. Dating and quantification of erosion processes based on exposed roots. Earth-Science Reviews, v. 123, p. 18-34, 2013. 
TOMLINS, G. Some Considerations in the Design of Low-Cost Remotely-Piloted Aircraft for Civil Remote Sensing Applications. Can. Surv. 1983, 37, 157-167.

TORRES SÁNCHEZ, J. et al. Configuration and specifications of an unmanned aerial vehicle (UAV) for early site specific weed management. PLoS One 8, e58210.Trans. ASAE 38, 259-269, 2003.

TREUHAFT, R.N.; LAW, B.E.; ASNER, G.P. Forest Attributes from Radar Interferometric Structure and Its Fusion with Optical Remote Sensing. BioScience 2004, 54, 561-571

WALKER, B., 1994. Global change extensive strategy agriculture options in the regions of the world. Clim. Change 27, 39 e 47.

WANG, Yuan et al. Estimating nitrogen status of rice using the image segmentation of G-R thresholding method. Field Crops Research, image segmantation mask out the effect of non-vegetation features,..., v. 149, p. 33-39, 2013

WISCHMEIER, W. H., \& SMITH, D. D. Predicting rainfall erosion losses-A guide to conservation planning. Predicting rainfall erosion losses. A guide to conservation planning, 1978

WOEBBECKE, D. M. et al. Color Indices for Weed Identification Under Various Soil, Residue, and Lighting Conditions. Transactions of the ASAE, v. 38, n. 1, p. 259-269, 1995.

YUAN, F. et al. Land cover classification and change analysis of the twin cities (Minnesota) metropolitan area by multitemporal Land sat remote sensing. Remote Sensing of Environment, v. 98, n. 2-3, p. 317-328, 2005.

ZHANG, J. et al. Estimating spatial variation in Alberta forest biomass from a combination of forest inventory and remote sensing data. Biogeosciences 11, 2793-2008, 2014.

ZHANG, J. et al. Regional and historical factors supplement current climate in shaping global forest canopy height. J. Ecol. 104, 469-478, 2016.

ZHANG, N.; WANG, M.; WANG, N. Precision agriculture - a worldwide overview. Computers and Electronics in Agriculture, v. 36, n. 2-3, p. 113-132, 2002. 